\section{OPEN ACCESS}

Edited by:

Ann E. Elsner,

Indiana University, United States

Reviewed by:

Vanessa Castelli,

University of L'Aquila, Italy

Paul Gamlin,

University of Alabama at Birmingham,

United States

*Correspondence:

Felipe P. Fiuza

felipe.fiuza@isd.org.br

Received: 01 October 2020 Accepted: 01 February 2021

Published: 24 February 2021

Citation:

Fiuza FP, Queiroz JPG, Aquino ACQ Câmara DA, Brandão LEM, Lima RH,

Cavalcanti JRLP, Engelberth RCGJ and Cavalcante JS (2021) Aging Alters Daily and Regional Calretinin Neuronal Expression in the Rat Non-image Forming Visual Thalamus. Front. Aging Neurosci. 13:613305 doi: 10.3389/fnagi.2021.613305

\title{
Aging Alters Daily and Regional Calretinin Neuronal Expression in the Rat Non-image Forming Visual Thalamus
}

\begin{abstract}
Felipe P. Fiuza ${ }^{1 *}$, José Pablo G. Queiroz ${ }^{1}$, Antônio Carlos Q. Aquino ${ }^{2}$, Diego A. Câmara ${ }^{2}$ Luiz Eduardo M. Brandão ${ }^{2,3}$, Ramon H. Lima ${ }^{1}$, José Rodolfo L. P. Cavalcanti ${ }^{4}$, Rovena Clara G. J. Engelberth ${ }^{2}$ and Jeferson S. Cavalcante ${ }^{2}$

1 Graduate Program in Neuroengineering, Edmond and Lily Safra International Institute of Neuroscience, Santos Dumont Institute, Macaiba, Brazil, ${ }^{2}$ Laboratory of Neurochemical Studies, Department of Physiology, Biosciences Center, Federal University of Rio Grande do Norte, Natal, Brazil, ${ }^{3}$ Department of Medical Sciences, Uppsala University, Uppsala, Sweden, ${ }^{4}$ Laboratory of Experimental Neurology, Department of Biomedical Sciences, Health Science Center, University of State of Rio Grande do Norte, Mossoró, Brazil
\end{abstract}

Aging affects the overall physiology, including the image-forming and non-image forming visual systems. Among the components of the latter, the thalamic retinorecipient inter-geniculate leaflet (IGL) and ventral lateral geniculate (vLGN) nucleus conveys light information to subcortical regions, adjusting visuomotor, and circadian functions. It is noteworthy that several visual related cells, such as neuronal subpopulations in the IGL and VLGN are neurochemically characterized by the presence of calcium binding proteins. Calretinin (CR), a representative of such proteins, denotes region-specificity in a temporal manner by variable day-night expression. In parallel, age-related brain dysfunction and neurodegeneration are associated with abnormal intracellular concentrations of calcium. Here, we investigated whether daily changes in the number of CR neurons are a feature of the aged IGL and VLGN in rats. To this end, we perfused rats, ranging from 3 to 24 months of age, within distinct phases of the day, namely zeitgeber times (ZTs). Then, we evaluated CR immunolabeling through design-based stereological cell estimation. We observed distinct daily rhythms of CR expression in the IGL and in both the retinorecipient (VLGNe) and non-retinorecipient (vLGNi) portions of the $v L G N$. In the ZT 6, the middle of the light phase, the CR cells are reduced with aging in the IGL and vLGNe. In the ZT 12, the transition between light to dark, an age-related CR loss was found in all nuclei. While CR expression predominates in specific spatial domains of vLGN, age-related changes appear not to be restricted at particular portions. No alterations were found in the dark/light transition or in the middle of the dark phase, ZTs 0 , and 18, respectively. These results are relevant in the understanding of how aging shifts the phenotype of visual related cells at topographically organized channels of visuomotor and circadian processing.

Keywords: aging, intergeniculate leaflet, ventral lateral geniculate nucleus, lateral geniculate body, calcium binding proteins, circadian rhythms, calretinin, stereology 


\section{INTRODUCTION}

Aging is characterized, for most living organisms, as a timedependent physiological decline associated with increases in mortality and decreases in fertility rates (Flatt and Partridge, 2018). A practical approach to understand how the aging phenotype is determined, as well as the relationship between normal aging and age-related pathologies, is to identify distinctive cellular alterations as hallmarks of the aging process (López-Otín et al., 2013). In the nervous system, for instance, despite there being few changes in the global neuronal numbers throughout life (Long et al., 1999; von Bartheld et al., 2016), neurochemical-specific subpopulations of cells are lost during aging (Bañuelos et al., 2013; Pal et al., 2019; Lamerand et al., 2020). Thus, given the diverse nature of brain neurochemistry, the characterization of age-related changes in a cellular level still poses a challenging endeavor for neuroscience.

There is clear evidence that age-related brain dysfunction and neurodegeneration are associated with abnormal intracellular concentrations of calcium $\left(\mathrm{Ca}^{2+}\right)$, as stated by the $\mathrm{Ca}^{2+}$ hypothesis of aging (Landfield, 1987; Khachaturian, 1994). Therefore, protein-mediated mechanisms of cytosolic $\mathrm{Ca}^{2+}$ buffering, such as the action of the EF-hand family of calcium binding proteins (CaBPs), may act as neuroprotective factors influencing age-related alterations (Alzheimer's Association Calcium Hypothesis Workgroup, 2017). Calretinin (CR), a representative of such CaBPs, seems to participate in the induction of long-term potentiation (Schurmans et al., 1997) and is present in neurons which are selectively resistant to the toxicity induced by $\beta$-amyloid protein, calcium overload, and excitatory amino acid stimulation in the brains of rodents (Lukas and Jones, 1994; Pike and Cotman, 1995). The CR and other CaBPs are often employed as histological markers due to their complementary distribution across brain regions and neuronal subclasses, being linked with region-specific vulnerability to aging effects (Fairless et al., 2019; Lamerand et al., 2020). Accordingly, the $\mathrm{CR}$ immunopositive $(\mathrm{CR}+)$ neurons are lost during aging in the cortical and subcortical regions of rodent and human brains (Villa et al., 1994; Bu et al., 2003; Bae et al., 2015; Ahn et al., 2017).

Aging affects the overall physiology, including visual, and circadian functions (Kondratova and Kondratov, 2012; Engelberth et al., 2013; Yan et al., 2020). After light reaches the retina, visual pathways to the brain nuclei are commonly grouped into image-forming and non-image forming visual systems (Lucas et al., 2014; Sondereker et al., 2020). A major retinorecipient zone lies in the thalamic lateral geniculate body, which is subdivided into dorsal lateral geniculate nucleus $(\mathrm{dLGN})$, intergeniculate leaflet (IGL), and ventral lateral

Abbreviations: asf, Area sampling fraction; CaBP, Calcium binding proteins; $\mathrm{CB}$, Calbindin d-28k; CE, Coefficient of error; CR, Calretinin; $\mathrm{CR}+$, Calretinin immunopositive; dLGN, Dorsal lateral geniculate nucleus; hsf, Height sampling fraction; IGL, Intergeniculate leaflet; opt, Optic tract; RGC, Retinal ganglion cell; SCN, Suprachiasmatic nucleus; ssf, Serial sampling fraction; vLGN, Ventral lateral geniculate nucleus; vLGNe, Ventral lateral geniculate nucleus external portion; vLGNi, Ventral lateral geniculate nucleus internal portion; ZT, Zeitgeber time; AU, Arbitrary units. geniculate nucleus (vLGN) (Morin and Studholme, 2014). Apart from the clear subdivision of the vLGN in an external retinorecipient (vLGNe) and an internal non-retinorecipient (vLGNi) portions, there is no obvious cytoarchitectonic differences in these regions (Monavarfeshani et al., 2017). Unlike the dLGN, the classical component of the image forming visual system, the IGL and vLGN have no thalamocortical relays to any cortical areas (Harrington, 1997; Monavarfeshani et al., 2017). Instead, the IGL and vLGN widely innervate subcortical visuomotor related nuclei, such as pretectal and optic accessory system regions, being likely the main thalamic source of afferents to the superior colliculus in rats (Matute and Streit, 1985; Harrington, 1997). Also, the IGL is known to provide photic and non-photic (e.g., novel or metabolic conditions) inputs to the hypothalamic suprachiasmatic nucleus (SCN) that finetunes the circadian photo-entrainment (Morin, 2013). For all these reasons, the IGL and vLGN are classified as the thalamic components of the non-image forming visual system (Fox and Guido, 2011; Chengetanai et al., 2020).

It is noteworthy that several visual-related nervous cells, such as the retinal ganglion cells (RGCs), SCN, IGL, and vLGN neurons are neurochemically characterized by the presence of CR (Arai et al., 1992; Jeon and Jeon, 1998; Lee et al., 2010a,b; Lee et al., 2016; Moore, 2016). Interestingly, the day-night variations in the CR expression denote region-specificity (Campos et al., 2015a,b). For instance, the rat SCN presents a higher density of $\mathrm{CR}+$ neurons in the light phase in comparison with the dark phase of the day (Moore, 2016). Notably, age-related alterations in the daily rhythmicity of other neurochemicals, such as serotonin, noradrenaline, dopamine, arginine vasopressin, and vasoactive intestinal polypeptide, are features of the brains of rodents and primates (Míguez et al., 1998; Cayetanot et al., 2005; Jagota and Kalyani, 2008). In fact, aging leads to a loss of rhythm in $90 \%$ of the hippocampal proteins that display circadian expression in mice (Adler et al., 2020). Thus, given the relationship among aging, calcium signaling, and circadian functions, one could hypothesize that daily changes in the CaBPs expression are markers of cellular aging and regional vulnerability. Here, we use unbiased stereology, the gold standard of cell quantification, to assess whether daily changes in the number of CR+ neurons occur in the IGL and vLGN of rats from different ages. In addition to presenting new data regarding this scarcely investigated issue, we discuss how the interpretation of morphometric data benefits from taking into consideration the chronobiologic context. Moreover, we present data supporting that a phase-dependent reduction in the amplitude of $\mathrm{CR}$ expression characterizes an age-related shift of neuronal phenotypes in the rat non-image forming visual thalamus.

\section{MATERIALS AND METHODS}

\section{Experimental Subjects}

A total of 48 male Wistar rats, ranging from 3 to 24 months of age, were housed in cages at $22^{\circ} \mathrm{C}$ and $50 \%$ humidity in a $12: 12 \mathrm{~h}$ light/dark cycle with food and water freely available. Animals were divided into four groups ( $n=12$ per group) regarding 
the photic condition, or zeitgeber, in which euthanasia was performed. By definition, the zeitgeber time (ZT) 12 corresponds to the lights-off hour in the animal housing facility. Specifically, animals were euthanized at ZT 0-dark/light transition, ZT 6-light phase, ZT 12-light/dark transition, and ZT 18- dark phase. All procedures were approved by local ethics committee (CEUAUFRN number 054/2015) in accordance with Brazilian law number 11.794/2008 for animal experimentation.

\section{Tissue Fixation}

Following anesthesia with sodium thiopental $(40 \mathrm{mg} / \mathrm{kg})$, animals were submitted for a thoracotomy and transcardiac perfusion through the left ventricle with a $300 \mathrm{ml} \mathrm{NaCl}$ solution (0.9\%) followed by $300 \mathrm{ml}$ formalin (10\%) in a $0.1 \mathrm{~m}$ phosphate buffer (PB, pH 7.4). These procedures were carried out in the beginning of each ZT. Following perfusion, the brains were removed, postfixed with the same fixative overnight and cryoprotected in a $30 \%$ sucrose solution with $0.1 \mathrm{~m} \mathrm{~PB} \mathrm{(pH} \mathrm{7.4)} \mathrm{for} 3$ days. Then, brains were sectioned into $50 \mu \mathrm{m}$ coronal slices in a cryostat (Leica Microsystems) and the sections were sequentially collected in 96well plates filled with antifreezing solution to be stored at $-20^{\circ} \mathrm{C}$ until use for immunohistochemistry. We further detailed tissue sampling under the Stereology topic.

\section{Immunohistochemistry}

Sections of IGL and vLGN were submitted to free-floating immunohistochemistry. Firstly, sections were blocked for endogenous peroxidase activity in $0.3 \%$ hydrogen peroxide. Then, sections were incubated overnight with rabbit anti-CR primary antibody (C7479, Sigma-Aldrich) in a 1:1,000 dilution with $2 \%$ bovine serum albumin and $0.1 \mathrm{~m}$ PB with $0.4 \%$ Triton X100 (PBTX 0.4\%). After rinsing, the sections were incubated with biotinylated goat anti-rabbit secondary antibody (111-065-003, Jackson ImmunoResearch) in a 1:1,000 dilution with PBTX 0.4\%. Then, sections were incubated in a $0.5 \%$ avidin-biotin solution (Vectastain standard ABC kit, PK-4000, Vector Laboratories), with $2.3 \% \mathrm{NaCl}$ addiction, for $120 \mathrm{~min}$. Finally, sections were placed with a $2.5 \%$ solution of diaminobenzidine (DAB) diluted with $0.1 \mathrm{~m} \mathrm{~PB}$. The final reaction was performed adding a $0.01 \% \mathrm{H}_{2} \mathrm{O}_{2}$ solution, to reveal brown-stained areas resulting from $\mathrm{DAB}$ oxidation. All sections were simultaneously removed from the $D A B$ solution after 2 min of reaction to minimize the staining variability. The brain sections were mounted on gelatinized slides, dried, dehydrated in graded ethanol solutions, cleared in xylene, and coverslipped with a DPX embedding matrix. Prior to histological processing, we performed a pilot study to establish optimal antibody concentration and incubation time. We confirmed internal positive controls by observing well-defined CR+ cell bodies in regions such as the cerebral cortex, the thalamic lateral posterior complex, and in the caudal zona incerta. Also, we addressed negative controls by incubating sections with no addition of primary antibody. In these cases, we observed no immunoreactivity.

\section{Stereology}

Unbiased stereological analyses were performed in an BX61 optical microscope (Olympus, Japan) fitted with a ProScan
II X-Y-Z motorized stage (Prior Scientific, Rockland, MA, United States), a $0.5 \mu \mathrm{m}$ resolution Heidenhain MT12 microcator (Heidenhain, Traunreut, Germany) and a Olympus DP-71 digital camera connected to a computer running the software NewCAST (Visiopharm, Hørsholm, Denmark). A pilot study was performed to establish optimal parameters of section fraction, counting frame area, grid-spacing size, and disector height for stereological analyses. Accordingly, we used a $1 / 4$ section sampling fraction (ssf), corresponding to $\sim 5$ IGL and 6 vLGN sections from an average of 24 sections identified with the aid of the seventh edition of the rat brain atlas in stereotaxic coordinates (Paxinos and Watson, 2014). We took into account, the anatomical considerations of Arai et al. (1992) and Harrington (1997), as well as previous observations of our group (Fiuza et al., 2016, 2017), to establish all stereological parameters and to trace the regional boundaries. Our systematic uniform random sampling was established by randomly choosing the first section and picking every following vLGN section in a $200 \mu \mathrm{m}$ interval.

\section{Regions of Interest}

In the lateral geniculate body of the rat, the optic tract (opt) carries out retinal projections to the dLGN, IGL, and vLGN laterally bordering these nuclei (Figure 1). The dorsal and medial borders of IGL, namely the dLGN and superior thalamic radiation, were easily identified since there is little or no $\mathrm{CR}$ staining in its adjacent boundaries. As the dorsal most regions of the vLGNe and vLGNi are relatively spared from CR+ neurons, the IGL ventral borders can be distinguished from its adjacent vLGN nuclei. At rostral levels, the vLGNe presents a stronger background of CR staining which serves as a clear division from vLGNi (Figure 1A). At the middle and caudal levels, vLGNe enlarges and this stronger background is more pronounced in its surroundings, rather than in the center, still delineating the vLGNe/vLGNi border (Figures 1B,C). Also, the vLGNe neurons usually present a stronger CR cytoplasmic signal which aids in nuclei delineation.

\section{Absolute Cell Number Estimation}

We estimated absolute CR + cell number bilaterally in an assumption-free manner for each nucleus following the optical fractionator method (West et al., 1991). Briefly, this methodology consists in overlaying a volumetric counting frame, the disector, in the histological material after the establishment of the ssf as well as area sampling fraction (asf) and height sampling fraction (hsf). The asf corresponds to the two-dimensional portion of the region of interest in which disectors are overlaid. The hsf comprehends the fraction of the $\mathrm{Z}$ plane that each disector probes. For our estimations, all samples were blind-coded so that the experimenter had no information regarding the age of the animal or its ZT group. First, we outlined the IGL and vLGN subdivisions with a low magnification objective (4x), and then counted the cells at high magnification (100x PlanApo oil lenses with 1.4 NA). We only counted the cells with the top of the nucleus clearly situated within the disector inclusion zone. Based on our pilot study, we determined a minimum of 100 counted cells to obtain reliable results with the coefficient of error (CE) 


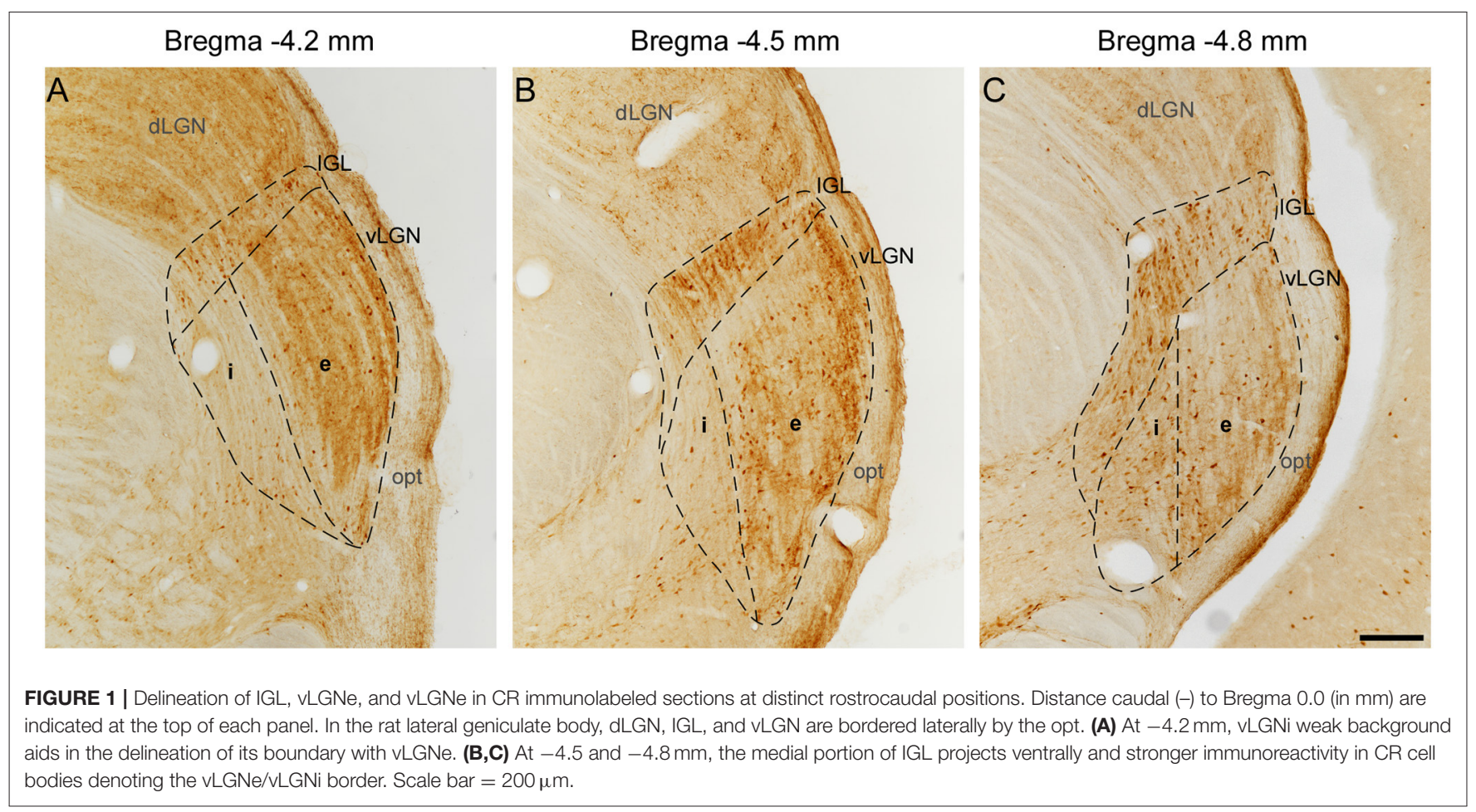

below 0.1. To achieve this minimum cell number for counting, we used a $50 \times 50 \mu \mathrm{m}$ counting frame with a grid spacing size of 90 $\times 90 \mu \mathrm{m}$ within our $1 / 4 \mathrm{ssf}$. We calculated the asf as a result of the counting frame area divided by grid-spacing area. The thickness of each post-shrinkage sections was calculated by focusing the topmost of tissue and slightly moving down to the bottommost focus point to measure the distance moved in the z-axis. As we observed that the tissue shrinks to an average value of $15 \mu \mathrm{m}$, the disector height was set at $12 \mu \mathrm{m}$ with $1.5 \mu \mathrm{m}$ guard zones above and below. Thus, the hsf was obtained through the division of the disector height by a number-weighted mean post-shrinkage section thickness. The CE was calculated with the smoothness class of $\mathrm{m}=1$ (Gundersen et al., 1999).

After obtaining cell counts $\left(Q^{-}\right)$from IGL and vLGN, the total number of cells $(N)$ was estimated by the optical fractionator equation (West et al., 1991):

$$
N=\sum Q^{-} \cdot \frac{1}{s s f} \cdot \frac{1}{a s f} \cdot \frac{1}{h s f}
$$

\section{Evaluation of CR Spatial Distribution}

We followed the approach of Sabbagh et al. (2020) to evaluate whether CR staining predominates particular vLGN portions in the lateral to medial axis at different ZTs. As there was no indication for the existence of any kind of such cellular organization in the IGL (Monavarfeshani et al., 2017), we did not evaluate this nucleus. For this evaluation, we developed a custom-line scan script that runs in the public domain software ImageJ FIJI version 1.52p (National Institutes of Health, USA). First, the image is calibrated into $\mu \mathrm{m}$ scale and the user contours the region of interest. Then, the image is transformed into 8-bit type, subtracted from the background and inverted, displaying the CR staining as pixel gray values. Finally, equally spaced lines are overlaid into the region of interest and plot profiles show the variation of pixel intensity along each line length. The data are plotted based on the average intensity of all lines within the section and every ssf section of the nucleus. Considering the variable stoichiometry in immunohistochemical procedures (Bishop et al., 2018), comparing intensities between the age and ZT groups could retrieve biased results. For this reason, we did not perform any direct statistical comparison with this dataset. Instead, we used its descriptive feature to evaluate the relative spatial predominance of CR staining in animals at earlier or later points of the lifespan.

\section{Statistical Analysis}

We performed a Kolmogorov-Smirnov test to assess the normality of our group distributions. After confirming that all distributions were normal, we computed Pearson's correlation coefficient and linear regression analysis to assess if there were any predictive relationships between age and CR cell numbers within each ZT group. Further, we divided our study population into three age groups, namely, young (3-7 months), middle-aged (12-18 months), and old (19-24 months) groups (Table 1). Then, we performed a two-way ANOVA followed by Tukey's test for post-hoc comparisons to compare the effects of age and ZT in CR cell numbers of each LGN nucleus. These analyses aided the identification of region-specific CR daily rhythmicity and to find whether the aging effects occurred in the earlier or later periods of animal lifespan. In all analyses, differences were considered significant at $p \leq 0.05$ and data are expressed as mean $\pm \mathrm{SD}$. 
TABLE 1 | Mean CR+ neuronal number estimated in the IGL, vLGNe, and VLGNi in each ZT and age groups.

\begin{tabular}{|c|c|c|c|c|}
\hline \multirow[t]{2}{*}{ ZT group } & \multirow{2}{*}{$\begin{array}{c}\text { Age group } \\
\text { (Mean } \pm \text { SD } \\
\text { months) }\end{array}$} & \multicolumn{3}{|c|}{ Nucleus } \\
\hline & & IGL & vLGNe & vLGNi \\
\hline \multirow[t]{3}{*}{0} & $\begin{array}{c}\text { Young } \\
(4 \pm 1.15)\end{array}$ & $1,889 \pm 516$ & $2,999 \pm 459$ & $1,916 \pm 375$ \\
\hline & $\begin{array}{c}\text { Middle-aged } \\
(16 \pm 2.70)\end{array}$ & $1,777 \pm 525$ & $2,634 \pm 721$ & $1,770 \pm 472$ \\
\hline & $\begin{array}{c}\text { Old } \\
(23.25 \pm 2.70)\end{array}$ & $1,950 \pm 443$ & $2,763 \pm 180$ & $1,713 \pm 695$ \\
\hline \multirow[t]{3}{*}{6} & $\begin{array}{c}\text { Young } \\
(4.5 \pm 1.91)\end{array}$ & $2,533 \pm 111$ & $4,055 \pm 619$ & $2,040 \pm 393$ \\
\hline & $\begin{array}{c}\text { Middle-aged } \\
(15 \pm 2.16)\end{array}$ & $2,279 \pm 421$ & $3,088 \pm 209^{\star}$ & $2,129 \pm 395$ \\
\hline & $\begin{array}{c}\text { Old } \\
(23.5 \pm 0.58)\end{array}$ & $1,963 \pm 240^{\star}$ & $3,163 \pm 218^{\star}$ & $1,729 \pm 430$ \\
\hline \multirow[t]{3}{*}{12} & $\begin{array}{c}\text { Young } \\
(4.75 \pm 1.70)\end{array}$ & $2,572 \pm 232$ & $3,480 \pm 256$ & $2,831 \pm 148$ \\
\hline & $\begin{array}{c}\text { Middle-aged } \\
(15.25 \pm 2.36)\end{array}$ & $2,397 \pm 170$ & $3,212 \pm 155$ & $2,415 \pm 459$ \\
\hline & $\begin{array}{c}\text { Old } \\
(22 \pm 1.41)\end{array}$ & $1,796 \pm 87^{\star \#}$ & $2,754 \pm 272^{\star}$ & $2,101 \pm 274^{\star}$ \\
\hline \multirow[t]{3}{*}{18} & $\begin{array}{c}\text { Young } \\
(3.75 \pm 0.95)\end{array}$ & $1,926 \pm 442$ & $2,909 \pm 702$ & $2,146 \pm 312$ \\
\hline & $\begin{array}{c}\text { Middle-aged } \\
(14 \pm 1.87)\end{array}$ & $1,874 \pm 203$ & $2,849 \pm 316$ & $1,901 \pm 472$ \\
\hline & $\begin{array}{c}\text { Old } \\
(20 \pm 1)\end{array}$ & $2,149 \pm 50$ & $3,393 \pm 245$ & $1,558 \pm 162$ \\
\hline
\end{tabular}

${ }^{*} p \leq 0.05$ in comparison with the young group within the same ZT group. \#p $\leq 0.05$ in comparison with the middle-aged group within the same ZT group. Data are expressed as mean $\pm S D$.

IGL, Intergeniculate leaflet; vLGNe, Ventral lateral geniculate nucleus external portion; vLGNi, Ventral lateral geniculate nucleus internal portion; ZT, Zeitgeber time.

Data analyses were performed with the GraphPad prism version 7.0 software.

\section{RESULTS}

\section{Daily Rhythmicity of CR Expression}

Representative IGL and vLGN CR immunolabeled sections of each ZT group in rats from distinct ages are shown in Figure 2. In Figure 3, we show $\mathrm{CR}+$ cells in higher magnification at $\mathrm{ZT}$ 12. After employing the two-way ANOVA with light condition as an isolated factor, we observed a significant effect upon $\mathrm{CR}$ neuronal changes in the IGL $\left[\mathrm{F}_{(3,36)}=4.07 ; p=0.01\right]$, vLGNe $\left[\mathrm{F}_{(3,36)}=4.82 ; p=0.006\right]$, and vLGNi $\left[\mathrm{F}_{(3,36)}=5.99\right.$; $p=0.002]$. In the IGL, Tukey's test for post-hoc comparisons revealed that young animals present a higher number of CR+ neurons at ZT $6(2,533 \pm 111)$, and ZT $12(2,572 \pm 232)$ in comparison with ZT $0(1,889 \pm 516)$. Also, we observed a significant reduction at the ZT $18(1,926 \pm 442)$ in comparison with ZT 12. In the vLGNe, Tukey's test for post-hoc comparisons revealed that young animals present a higher number of $\mathrm{CR}+$ neurons at ZT $6(4,055 \pm 619)$ in comparison with ZT $0(2,999$ $\pm 459)$ and ZT $18(2,909 \pm 702)$. In the vLGNi, Tukey's test for post-hoc comparisons revealed that young animals present a higher number of CR+ neurons at the ZT $12(2,831 \pm 148)$ in comparison with ZT 0 (1,916 \pm 375$)$ and ZT 6 (2,040 \pm 393$)$. In middle-aged and old animals, we observed no alterations in CR numbers at different ZTs in any nucleus (Figure 4).

\section{Age-Related Changes in CR Cell Numbers of IGL and VLGN}

Mean IGL, vLGNe, and vLGNi CR cell numbers of each age and ZT group are summarized in Table 1. In the IGL, two-way ANOVA with age as an isolated factor revealed no significant effects upon CR cell number alterations $\left[\mathrm{F}_{(2,36)}=2.44 ; p=0.10\right]$. However, we found a significant effect between the interaction of age and ZT factors in the changes of CR cell numbers $\left[\mathrm{F}_{(6,36)}\right.$ $=2.334 ; p=0.05]$. In the ZT 6 , the Tukey's test for post-hoc comparisons revealed a significant CR cell number reduction in the old animals $(1,963 \pm 240)$ in comparison with the young ones $(2,533 \pm 111)$. In the $\mathrm{ZT} 12$, we found a significant reduction of CR cells in old rats $(1,796 \pm 87)$ in comparison with both young $(2,572 \pm 232)$, and middle-aged $(2,397 \pm 170)$ groups (Figure 4A).

In the vLGNe, we observed CR cell numbers changed due to age factor $\left[\mathrm{F}_{(2,36)}=4.6 ; p=0.01\right]$ and the interaction between age and ZT factors $\left[\mathrm{F}_{(6,36)}=2.51 ; p=0.04\right]$. In the ZT 6, Tukey's post-hoc test revealed a reduction in the CR cell numbers for the old $(3,163 \pm 218)$ and middle-aged $(3,088 \pm 209)$ groups in comparison with the young ones $(4,055 \pm 619)$. In the ZT 12 , Tukey's post-hoc test revealed a significant reduction in CR cell numbers in the old $(2,754 \pm 272)$ animals in comparison with the young $(3,480 \pm 256)$ group (Figure 4B).

In the vLGNi, we observed CR cell numbers changed due to age factor $\left[\mathrm{F}_{(2,36)}=4.73 ; p=0.01\right]$ but the interaction between age and ZT factors had no significant effects $\left[\mathrm{F}_{(6,36)}=0.48 ; p=\right.$ 0.8 ]. In the ZT 12, Tukey's post-hoc test revealed a reduction in CR cell numbers of old $(2,101 \pm 274)$ rats in comparison with the young ones $(2,831 \pm 148)$ (Figure $4 \mathrm{C})$.

Considering age as a continuous variable, we reported negative correlations between age and CR cell numbers in IGL $(r=-0.72, p=0.008)$ and vLGNe $(r=-0.63, p=0.03)$ at the ZT 6. Also, we found negative correlations between age and CR cell numbers in IGL $(r=-0.81, p=0.002)$, vLGNe $(r=-0.79$, $p=0.002)$, and vLGNi at the ZT $12(r=-0.73, p=0.007)$. We observed no correlations between these two variables in any nucleus in the other ZT groups (Figure 5).

\section{Spatial Distribution of CR in the vLGN}

Through line scan analysis, we distinguished four spatial vLGNe and one vLGNi domains marked by CR staining (Figure 6). We refer to those as e1, e2, e3, e4, and vLGNi zones. As different animals have slight differences in vLGN length, here we use approximations of average distances we found for the young group. From the lateral-most border to $50 \mu \mathrm{m}$, we observed that the e1 zone is relatively spared from CR (young: $16.34 \pm 4.40$; old: $14.36 \pm 4.05)$. In young animals at the ZT 6, however, we found a higher predominance of CR staining in this area $(35.17 \pm 11.11)$. Between e 1 and $190 \mu \mathrm{m}$, we observed the e $2 \mathrm{CR}$ rich area (young: $24.74 \pm 5.15$; old: $21.55 \pm 3.69$ ). Between e2 and $350 \mu \mathrm{m}$, we 


\section{Zeitgeber Time}

0
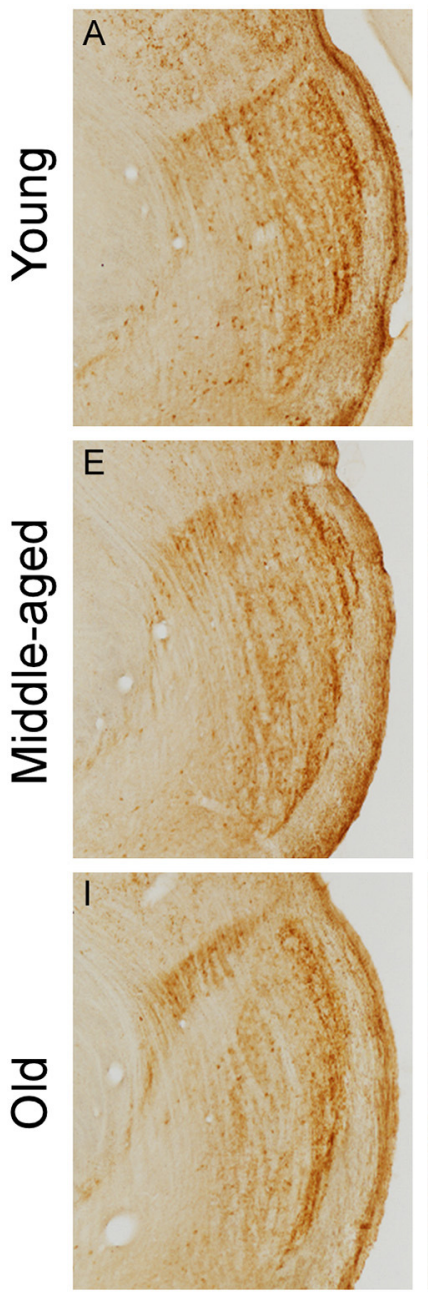

6
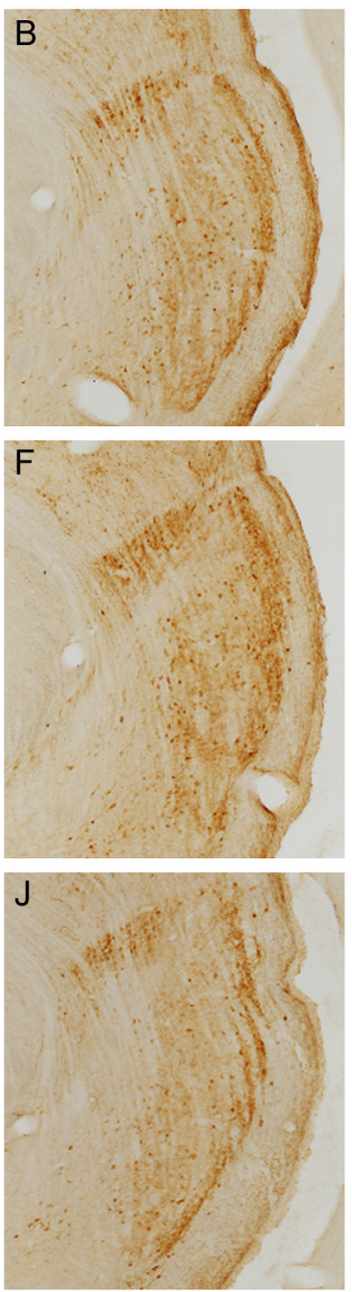

12
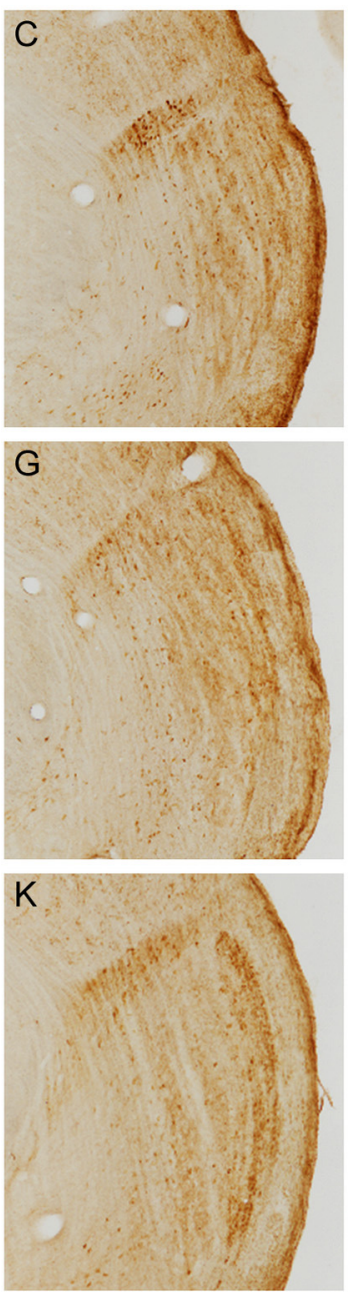

18
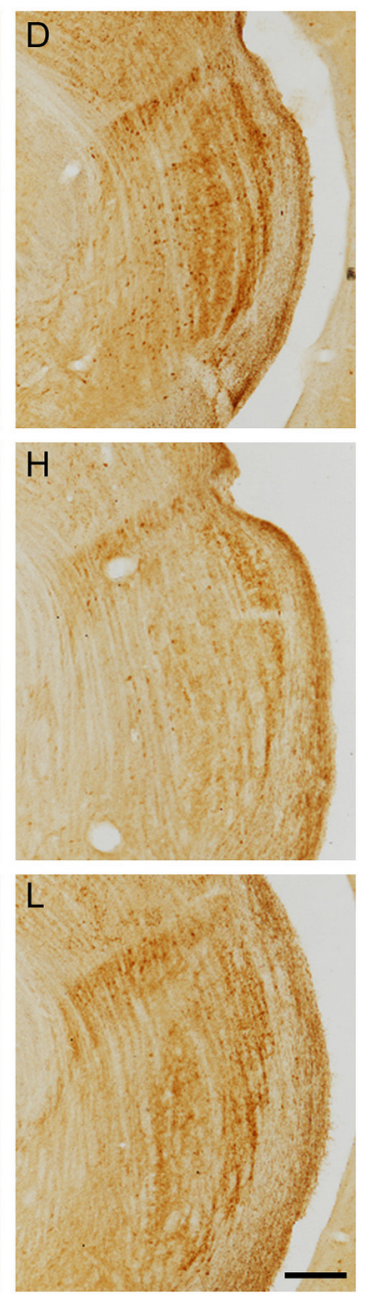

FIGURE 2 | Photomicrographs of CR immunolabeled histological sections containing the IGL, vLGNe, and vLGNi in animals from distinct age and ZT groups. CR sections are obtained after brain perfusion at ZTs 0 (dark/light phase), 6 (light phase), 12 (light/dark phase), and 18 (dark phase) from young (A-D), middle-aged (E-H), or old (I-L) animals. Scale bar $=225 \mu \mathrm{m}$.

found another CR-scarce zone that we refer as e3 (young: 16.54 \pm 1.98 ; old: $14.93 \pm 2.47$ ). Between e3 and $420 \mu \mathrm{m}$, we report the $4 \mathrm{CR}$ rich area highlighting the medial-most vLGNe border with vLGNi (young: $20.49 \pm 4.36$; old: $17.09 \pm 4.04$ ). From e4 to the medial-most boundary of vLGNi, we observed no particular pattern of CR staining (young: $11.62 \pm 4.48$; old: $10.20 \pm 3.84$ ). Data for CR intensity in each age group are presented here as the average of all ZTs, except for the e1 in which ZT 6 was isolated.

\section{DISCUSSION}

In this study, we describe how changes in the daily expression of the calcium binding protein, $\mathrm{CR}$, marks region-specific patterns of cellular aging in the rat non-image forming visual thalamus.
In a previous work, we quantified the total neuronal number for each of the subregions analyzed here using the same agegroups (Fiuza et al., 2017). Comparing these datasets, we can infer CR+ neurons correspond to $32-45 \%$ of the total neuronal population in the IGL, $16-23 \%$ in the vLGNe, and $13-20 \%$ in the vLGNi of rats. Considering CR is a known marker for GABAergic interneurons in other regions such as the cerebral cortex and amygdaloid complex (Tremblay et al., 2016; García-Amado and Prensa, 2021), it is likely that a sizeable portion of these neurons also composes GABAergic neuronal subpopulations. In fact, almost the entirety, if not all, of IGL cells are immunopositive for GAD, an enzyme that synthesizes GABA (Moore and Speh, 1993; Langel et al., 2018). Also, Gad1 and Gad2 mRNA, which translates the GAD 67 and GAD 65 isoforms, are expressed by 


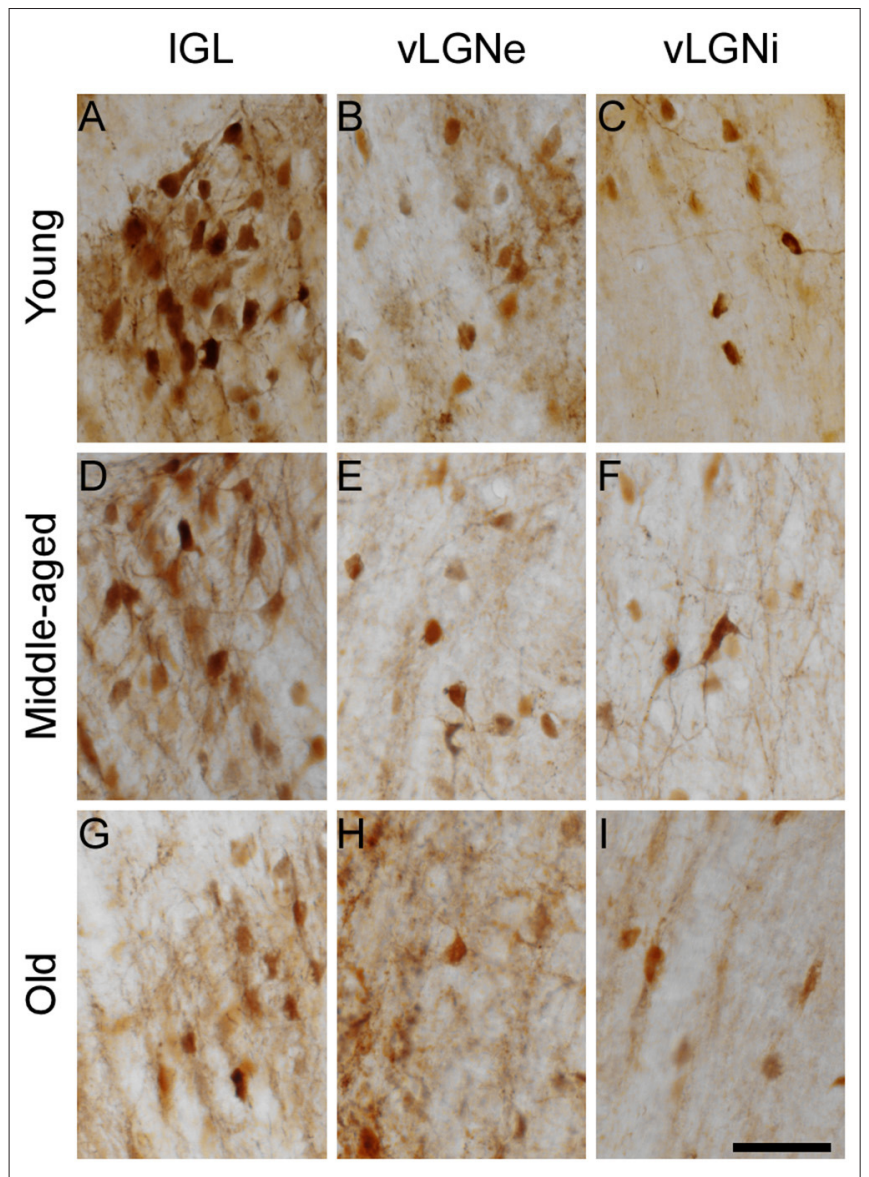

FIGURE 3 | High-magnification photomicrographs of CR immunolabeled cells of the IGL, VLGNe, and vLGNi at ZT 12. The IGL, VLGNe, and VLGNiCR+ neurons from young $(\mathbf{A}-\mathbf{C})$, middle-aged (D-F), and old (G-I) animals are shown in detail. Scale bar $=50 \mu \mathrm{m}$.

25 and $41 \%$, respectively, of the total DAPI stained cells in the mouse vLGN (Sabbagh et al., 2020). It is important to highlight that $\mathrm{CR}+$ neurons are also present in the geniculate body of lizard (Dávila et al., 2000), African wild dog (Chengetanai et al., 2020), banded mongoose, ferret (Pillay et al., 2020), marmoset, rock cavy (Cavalcante et al., 2008), and human brains (Münkle et al., 2000) denoting this characteristic is conserved, at some extent, during evolution. Since the IGL and vLGN contribute to visuomotor and circadian functions through projections to the superior colliculus, the pretectal nuclei, and suprachiasmatic nucleus in many species (Harrington, 1997; Morin, 2013), our findings could bring an interesting perspective for further translational aging studies.

We observed a phase-dependent CR expression in the IGL, vLGNe, and vLGNi of young rats. While every nucleus presented a higher number of CR cells during the light phase of the day, distinct phase-dependent patterns denoted a regional variability. In the IGL, we estimated higher numbers of CR + cells in both ZTs 6 and 12 in comparison with ZT 18. However, CR+ cells peak only at ZT 6 in vLGNe and only at ZT 12 in vLGNi. Such a daily
A

IGL

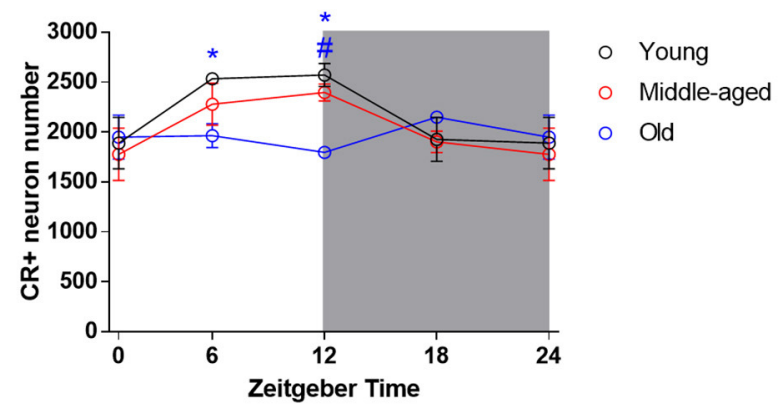

B

vLGNe

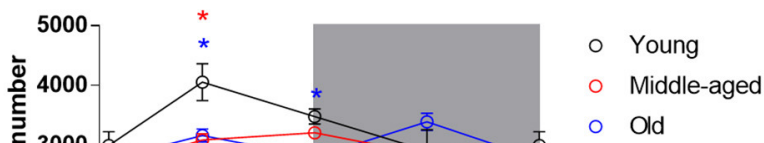

C

vLGNi

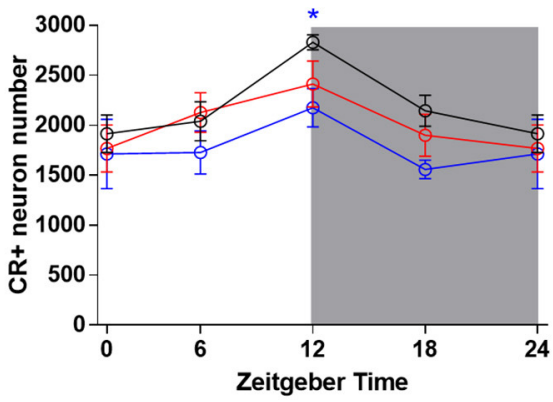

○ Young

○ Middle-aged

O Old

FIGURE 4 | Daily rhythmicity of CR expression in the IGL, VLGNe, and VLGNi. Stereological estimations of total $C R$ cell number at each ZT in the IGL (A), VLGNe (B), and VLGNi (C) from young (black), middle-aged (red), or old (blue) animals. Data from ZT 0 are double plotted as ZT 24, to graphically represent the $24 \mathrm{~h}$ of the day. Shaded gray areas represent the dark phase. Blue ${ }^{*} p<0.05$ in young vs. old comparison. Blue $\# p<0.05$ in middle-aged vs. old comparison. Red ${ }^{*} p<0.05$ in young vs. old comparison. Data are analyzed by two-way ANOVA followed by Tukey's test for post-hoc comparison. Data are plotted as mean $\pm \mathrm{SD}$.

rhythmicity of CR expression, with higher CR+ counts at ZT 6 in comparison with ZTs 14 and 19, is also a feature of a neuronal subpopulation found in the rat suprachiasmatic nucleus (Moore, 2016). Additionally, in Sapajus apella, a diurnal primate, a similar 
Fiuza et al.

Aging Alters Thalamic Calretinin Expression

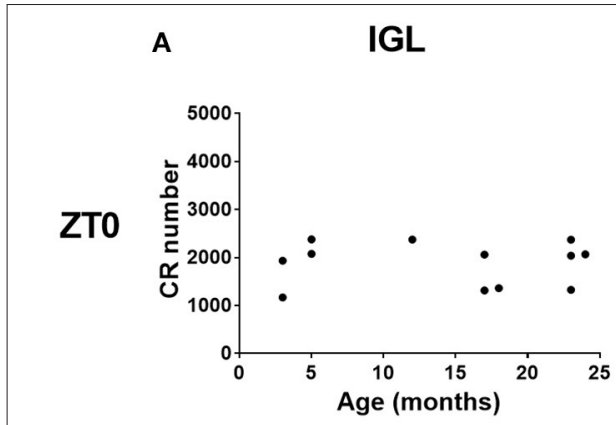

B

ZT6

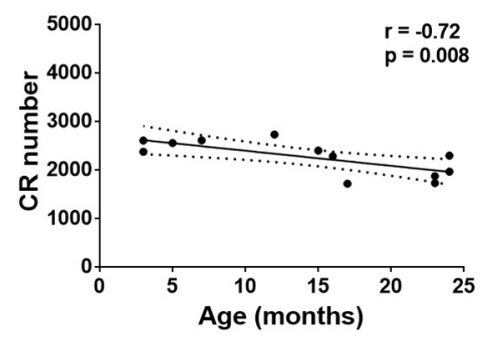

C

ZT12

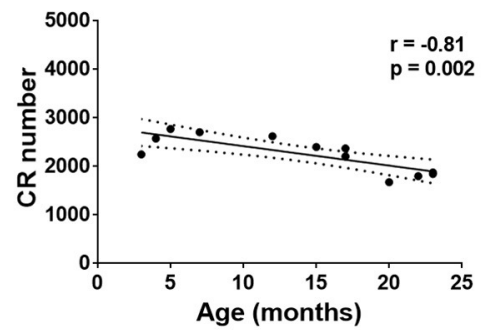

D

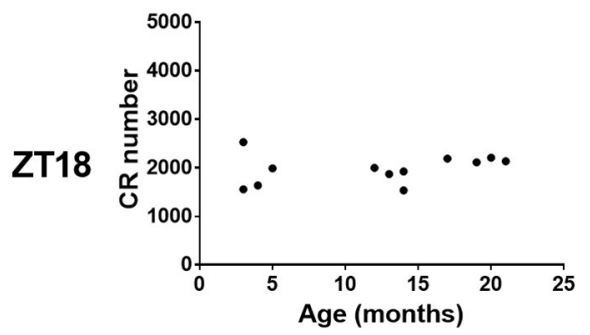

E

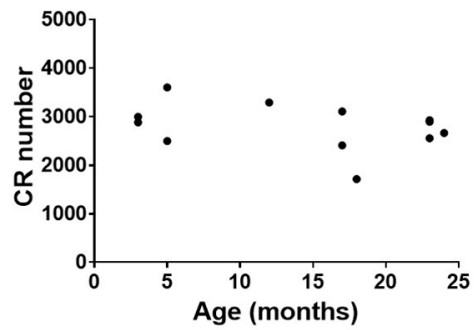

F

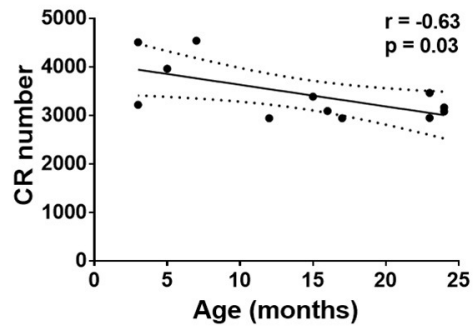

G

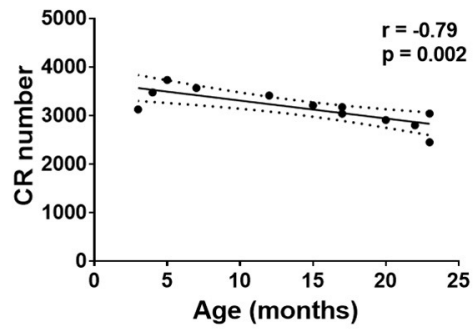

H

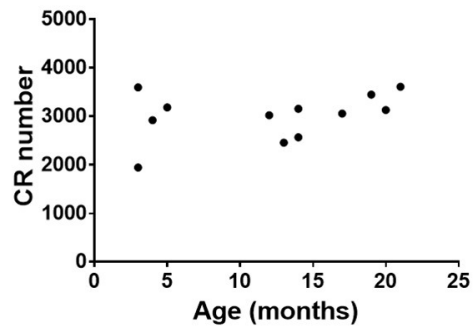

vLGNi

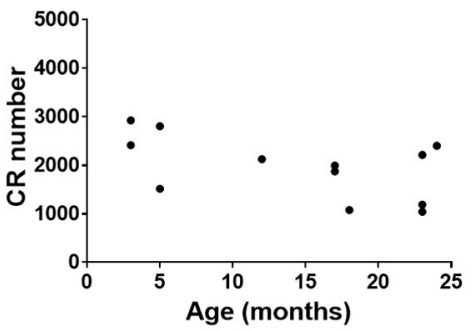

J

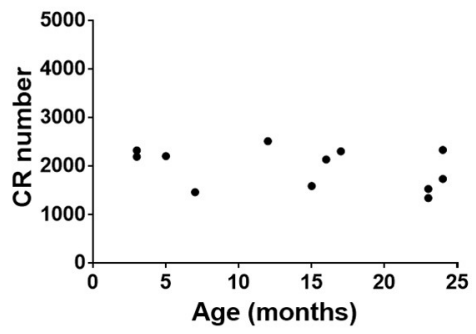

K

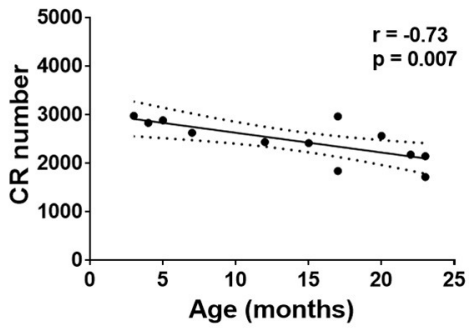

L

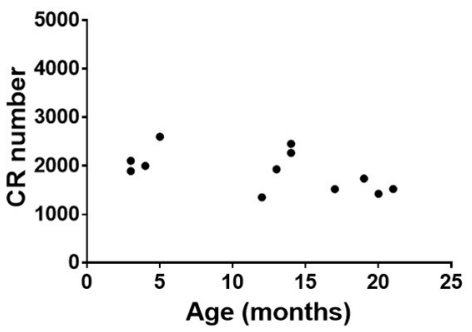

FIGURE 5 | Age-related changes in the CR expression of the IGL, vLGNe, and vLGNi. Total CR+ cell numbers of the IGL (A-D), vLGNe (E-H), and vLGNi (I-L) are plotted as a function of age in each ZT. Pearson's correlation coefficient $(r)$, regression lines with $95 \%$ confidence intervals and $p$-values are shown when a significant linear relation is observed.

Frontiers in Aging Neuroscience | www.frontiersin.org

8

February 2021 | Volume 13 | Article 613305 

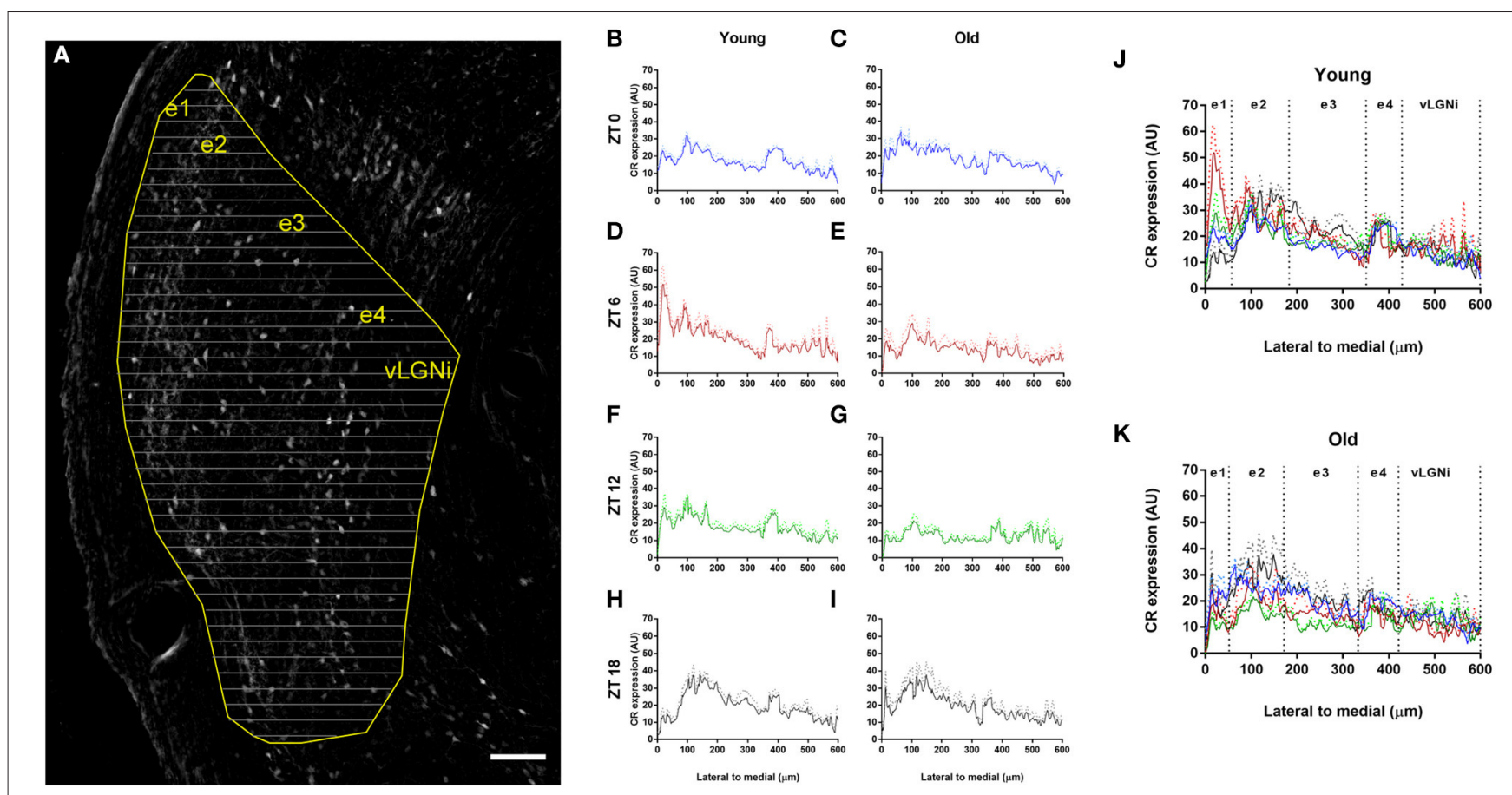

$\mathbf{F}$
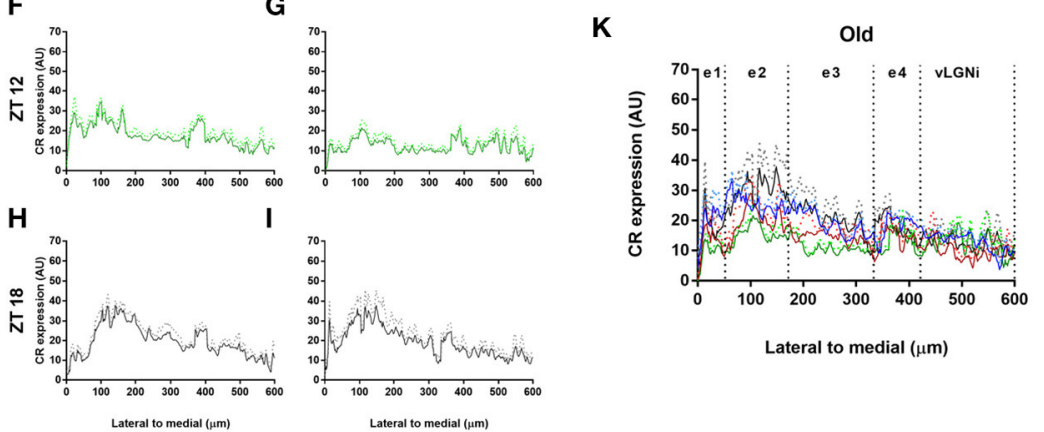

FIGURE 6 | Spatial distribution of CR staining in the VLGN. (A) Photomicrograph of the vLGN digitally processed for background subtraction. The vLGN borders are drawn (yellow) and equally spaced lines (gray) in the lateral to medial axis are overlaid to plot profiles of pixel intensity variations in arbitrary units (AU). Scale bar = $100 \mu \mathrm{m}$. (B-I) CR staining levels through the lateral to medial axis in the young and old animals at t ZTs 0 (blue), 6 (red), 12 (green), and 18 (black). (J,K) Every ZT plot overlaid for the young and old animals. Relative CR predominance distinguishes five zones, namely, e1, e2, e3, e4, and vLGNi domains. Data are plotted as mean gray values with standard error for each group.

pattern of reduced CR expression in the dark phase occurs in the ventral and dorsal subdivisions of the medial geniculate body and in the granular cell layer, but not in the polymorphic layer, of the dentate gyrus (Campos et al., 2015a,b). It is also noteworthy that, contrary to what we found for $\mathrm{CR}$, no daily rhythms were detected for the CaBP calbindin D-28k (CB) in the adult rat IGL (Arvanitogiannis et al., 2000). Therefore, it is conceivable that CB and CR characterize distinct neuronal subpopulations in the IGL as occurs in the rat arcuate nucleus and in the human temporal cortex (del Río and DeFelipe, 1996; Foo et al., 2014).

Although daily rhythms of $\mathrm{CR}$ expression might reflect important intracellular mechanisms of calcium buffering and signaling, we showed here that it progressively declines with age in the rat non-image forming thalamus. In fact, middleaged and old animals were completely absent of daily variations in the CR + cell number of every nucleus. To the best of our knowledge, only one other study addressed the question of whether daily-rhythms of CaBP expression could be a hallmark of cellular aging. In this study, no daily or age-related changes in the density of CB immunopositive neurons were observed in the SCN of the primate, Microcebus murinus (Cayetanot et al., 2007). Here, we highlight time of the day as an important methodological consideration for CaBP cell number estimation as two independent studies carried out at isolated ZTs, for instance, one at ZT 12 and other at ZT18, could report either age-related neuronal loss or no alterations whatsoever.
Treating age as a continuous variable, we observed age-related reduction in $\mathrm{CR}+$ neurons in all three nuclei at the $\mathrm{ZT} 12$. At ZT 6 , we found such alterations only in IGL and vLGNe. No changes were found in any nucleus at the other ZTs. On comparing with the age categories dataset, such neuronal loss seems to occur at different rates. At the ZT 6 we can detect changes in CR+ neurons of vLGNe in middle-aged rats whereas in the other nuclei at ZT 6 and in every nucleus at ZT 12, the cell numbers were altered only in the old animals. Considering the total neuronal number of these regions remains stable during aging (Fiuza et al., 2017), our findings here are likely due to loss in the capacity of neurons to express CR rather than death of CR+ neuronal population with aging. Such findings add up to a number of reports regarding age-related reductions in $\mathrm{CR}+$ neurons in the hippocampus (Villa et al., 1994), inferior colliculus (Ouda et al., 2012), striatum (Bae et al., 2015), and somatosensory cortex (Ahn et al., 2017) of rodent brains, as well as in the temporal and auditory regions of the human cerebral cortex (Bu et al., 2003). Age-related pathologies also result in decreased number of $\mathrm{CR}+$ neuronal population, as observed in the olfactory cortex of transgenic A $\beta$ PP/PS1 mice (Saiz-Sanchez et al., 2012) and in the pyriform cortex of patients with Alzheimer's disease (SaizSanchez et al., 2015). In some scenarios, these alterations might be due to a specific reduction of $\mathrm{CR}$ expression rather than cell death as we observed here. For instance, the number of $\mathrm{CR}+$ large striatal interneurons, but not the absolute number of 
these cells, is decreased in Huntington's disease (Massouh et al., 2008). Notwithstanding, in the rat dorsomedial and ventromedial hypothalamic nuclei the percentage of $\mathrm{CR}+$ neurons increases during aging (Vishnyakova et al., 2020). This reinforces the usefulness in understanding $\mathrm{CR}$, or any $\mathrm{CaBP}$, expression as regional markers of cellular changes in normal aging and in the progression of age-related diseases.

Through our analysis of spatial distribution, we observed a predominance of CR staining into the lateral-most and medialmost portions of the vLGNe, with a low density CR zone between these regions, at all ZTs. In the vLGNi, however, we distinguished no specific pattern of CR distribution. That was the case even in the old animals, suggesting that the age-related cell loss we observed is not restricted at a particular subset of spatiallylocated neurons, except perhaps in the e1 subdivision at ZT 6 . We show that CR fairly delimits the vLGNe since sublaminaespecific markers reveal four hidden laminae, all containing GABAergic cells, in a lateral to medial organization (Sabbagh et al., 2020). It is noteworthy that in the vLGNe, but not in the IGL, retinal terminals are segregated into non-overlapping eye domains (Monavarfeshani et al., 2017). Such retinal projections to these nuclei arise from non-image forming RGCs, such as the M1 intrinsically photosensitive and Cdh3-GFP RGCs (Hattar et al., 2006; Osterhout et al., 2011). The lateral-most regions of the vLGNe, which we relate with CR delimited e1, presents retinal terminals from on-off direction-selective RGCs (Monavarfeshani et al., 2017). Also, rods and cones seem to participate in the IGL/vLGN activation since light-induced FOS immunoreactivity is reduced in these nuclei of mice lacking these photoreceptors, a response even more dampened in the aged animals (Lupi et al., 2012). Considering CR+ neurons in the IGL/vLGN may be targets from CR expressing RGCs (Arai et al., 1992), further works are necessary to establish whether a coincident loss of CR expression denotes age-related effects on topographically organized channels of visuomotor and circadian processing.

Unbiased stereological methods for quantitative neuroanatomy greatly enriched the knowledge of how aging affects the nervous system by directing a region-specific approach to detect age-related alterations in the cellular composition of the brain. As many studies focus in total protein quantification to address age-related changes, we highlight the informative feature to quantify cell numbers for an integrative approach. For instance, in here and in a few previous reports, if any, the $\mathrm{CR}+$ cell bodies were found in the rodent dLGN (Arai et al.,

\section{REFERENCES}

Adler, P., Chiang, C. K., Mayne, J., Ning, Z., Zhang, X., Xu, B., et al. (2020). Aging disrupts the circadian patterns of protein expression in the murine hippocampus. Front Aging Neurosci. 11:368. doi: 10.3389/fnagi.2019. 00368

Ahn, J. H., Hong, S., Park, J. H., Kim, I. H., Cho, J. H., Lee, T. K., et al. (2017). Immunoreactivities of calbindin-D28k, calretinin and parvalbumin in the somatosensory cortex of rodents during normal aging. Mol. Med. Rep. 16, 7191-7198. doi: 10.3892/mmr.2017.7573

Alzheimer's Association Calcium Hypothesis Workgroup. (2017). Calcium hypothesis of Alzheimer's disease and brain aging: a framework for integrating
1992; Evangelio et al., 2018). However, in comparison with IGL and vLGN, the mouse dLGN presents similar CR levels detected by Western blot and even higher expression of its associated Calb 2 mRNA (Sabbagh et al., 2018). While mRNA levels are not necessarily correlated with protein amounts (Maier et al., 2009), such findings might be due to the presence of CR in axon terminals at the dLGN as it occurs in the macaque prefrontal cortex (Melchitzky et al., 2005). Thus, mRNA and total protein expression approaches could benefit from the understanding if potential changes in molecule amount are accompanied by shifts in the cellular phenotype.

\section{DATA AVAILABILITY STATEMENT}

The raw data supporting the conclusions of this article will be made available by the authors, without undue reservation.

\section{ETHICS STATEMENT}

The animal study was reviewed and approved by Comissão de ética no uso de animais (CEUA/UFRN).

\section{AUTHOR CONTRIBUTIONS}

FF, RE, and JC designed the study and wrote the paper receiving inputs from all the authors. FF, AA, DC, LB, and JC carried out histological procedures. FF, JQ, and RL performed image analysis and prepared figures. All authors contributed to the article and approved the submitted version.

\section{FUNDING}

This work was supported by the Brazilian funding agencies, Conselho Nacional de Desenvolvimento Científico e Tecnológico (CNPQ), and Coordenação de Aperfeiçoamento de Pessoal de Ensino Superior (CAPES).

\section{ACKNOWLEDGMENTS}

We thank Profs. Carlos Avendaño and Francisco Clascá from the Universidad Autonoma de Madrid-Spain for aiding in the stereological and microscopy analyses. new evidence into a comprehensive theory of pathogenesis. Alzheimers Dement. 13, 178-82.e17. doi: 10.1016/j.jalz.2016.12.006

Arai, M., Arai, R., Kani, K., and Jacobowitz, D. M. (1992). Immunohistochemical localization of calretinin in the rat lateral geniculate nucleus and its retino-geniculate projection. Brain Res. 596, 215-222. doi: 10.1016/0006-8993(92)91550-X

Arvanitogiannis, A., Robinson, B., Beaulé, C., and Amir, S. (2000). Calbindin-D28k immunoreactivity in the suprachiasmatic nucleus and the circadian response to constant light in the rat. Neuroscience 99, 397-401. doi: 10.1016/S0306-4522(00)00327-4

Bae, E. J., Chen, B. H., Shin, B. N., Cho, J. H., Kim, I. H., Park, J. H., et al. (2015). Comparison of immunoreactivities of calbindin-D28k, calretinin and 
parvalbumin in the striatum between young, adult and aged mice, rats and gerbils. Neurochem. Res. 40, 864-872. doi: 10.1007/s11064-015-1537-x

Bañuelos, C., LaSarge, C. L., McQuail, J. A., Hartman, J. J., Gilbert, R. J., Ormerod, B. K., et al. (2013). Age-related changes in rostral basal forebrain cholinergic and GABAergic projection neurons: relationship with spatial impairment. Neurobiol. Aging 34, 845-862. doi: 10.1016/j.neurobiolaging.2012.06.013

Bishop, D. P., Cole, N., Zhang, T., Doble, P. A., and Hare, D. J. (2018). A guide to integrating immunohistochemistry and chemical imaging. Chem. Soc. Rev. 47, 3770-3787. doi: 10.1039/C7CS00610A

Bu, J., Sathyendra, V., Nagykery, N., and Geula, C. (2003). Age-related changes in calbindin-D28k, calretinin, and parvalbumin-immunoreactive neurons in the human cerebral cortex. Exp. Neurol. 182, 220-231. doi: 10.1016/S0014-4886(03)00094-3

Campos, L. M. G., Cruz-Rizzolo, R. J., and Pinato, L. (2015a). The primate seahorse rhythm. Brain Res. 1613, 81-91. doi: 10.1016/j.brainres.2015.03.054

Campos, L. M. G., Osório, E. C., Santos, G. L., da, S., Nogueira, M. I., Cruz-Rizzolo, R. J., et al. (2015b). Temporal changes in calcium-binding proteins in the medial geniculate nucleus of the monkey Sapajusapella. J. Chem. Neuroanat. 68, 45-54. doi: 10.1016/j.jchemneu.2015.07.005

Cavalcante, J. S., Britto, L. R. G., Toledo, C. A. B., Nascimento, E. S., Lima, R. R. M., Pontes, A. L. B., et al. (2008). Calcium-binding proteins in the circadian centers of the common marmoset (Callithrix jacchus) and the rock cavy (Kerodon rupestris) brains. Brain Res. Bull. 76, 354-360. doi: 10.1016/j.brainresbull.2008.02.028

Cayetanot, F., Bentivoglio, M., and Aujard, F. (2005). Arginine-vasopressin and vasointestinal polypeptide rhythms in the suprachiasmatic nucleus of the mouse lemur reveal aging-related alterations of circadian pacemaker neurons in a non-human primate. Eur. J. Neurosci. 22, 902-910. doi: 10.1111/j.1460-9568.2005.04268.x

Cayetanot, F., Deprez, J., and Aujard, F. (2007). Calbindin D28K protein cells in a primate suprachiasmatic nucleus: localization, daily rhythm and age-related changes. Eur. J. Neurosci. 26, 2025-2032. doi: 10.1111/j.1460-9568.2007.05826.x

Chengetanai, S., Bhagwandin, A., Bertelsen, M. F., Hård, T., Hof, P. R., Spocter, M. A., et al. (2020). The brain of the African wild dog. IV. The visual system. J. Comp. Neurol. 528, 3262-84. doi: 10.1002/cne.25000

Dávila, J. C., Guirado, S., and Puelles, L. (2000). Expression of calciumbinding proteins in the diencephalon of the lizard Psammodromus algirus. J. Comp. Neurol. 427, 67-92. doi: 10.1002/1096-9861(20001106)427:1<67::AIDCNE5 $>3.0 . \mathrm{CO} ; 2-2$

del Río, M. R., and DeFelipe, J. (1996). Colocalization of calbindin D$28 \mathrm{k}$, calretinin, and GABA immunoreactivities in neurons of the human temporal cortex. J. Comp. Neurol. 369, 472-482. doi: 10.1002/(SICI)10969861(19960603)369:3<472::AID-CNE11>3.0.CO;2-K

Engelberth, R. C. G. J., Pontes, A. L. B., de, Fiuza, F. P., Silva, K. D., de, A., Resende, N. da, S., et al. (2013). Changes in the suprachiasmatic nucleus during aging: implications for biological rhythms. Psychol. Neurosci. 6, 287-297. doi: 10.3922/j.psns.2013.3.07

Evangelio, M., García-Amado, M., and Clascá, F. (2018). Thalamocortical projection neuron and interneuron numbers in the visual thalamic nuclei of the adult C57BL/6 mouse. Front. Neuroanat. 12:27. doi: 10.3389/fnana.2018. 00027

Fairless, R., Williams, S. K., and Diem, R. (2019). Calcium-binding proteins as determinants of central nervous system neuronal vulnerability to disease. Int. J. Mol. Sci. 20:2146. doi: 10.3390/ijms20092146

Fiuza, F. P., Aquino, A. C. Q., Câmara, D. A., Cavalcanti, J. R. L. P., Nascimento Júnior, E. S., Lima, R. H., et al. (2017). Region-specific glial hyperplasia and neuronal stability of rat lateral geniculate nucleus during aging. Exp. Gerontol. 100, 91-99. doi: 10.1016/j.exger.2017.11.001

Fiuza, F. P., Silva, K. D. A., Pessoa, R. A., Pontes, A. L. B., Cavalcanti, R. L. P., Pires, R. S., et al. (2016). Age-related changes in neurochemical components and retinal projections of rat intergeniculate leaflet. Age 38:4. doi: 10.1007/s11357-015-9867-9

Flatt, T., and Partridge, L. (2018). Horizons in the evolution of aging. BMC Biol. 16:93. doi: 10.1186/s12915-018-0562-Z

Foo, K. S., Hellysaz, A., and Broberger, C. (2014). Expression and colocalization patterns of calbindin-D28k, calretinin and parvalbumin in the rat hypothalamic arcuate nucleus. J. Chem. Neuroanat. 61-62, 20-32. doi: $10.1016 /$ j.jchemneu.2014.06.008
Fox, M. A., and Guido, W. (2011). Shedding light on class-specific wiring: development of intrinsically photosensitive retinal ganglion cell circuitry. Mol. Neurobiol. 44, 321-329. doi: 10.1007/s12035-011-8199-8

García-Amado, M., and Prensa, L. (2021). Neurons expressing parvalbumin and calretinin in the human amygdaloid complex: a quantitative and qualitative analysis in every nucleus and nuclear subdivision. Neuroscience 452, 153-168. doi: 10.1016/j.neuroscience.2020.11.001

Gundersen, H. J., Jensen, E. B., Kiêu, K., and Nielsen, J., null (1999). The efficiency of systematic sampling in stereology-reconsidered. J. Microsc. 193, 199-211. doi: 10.1046/j.1365-2818.1999.00457.x

Harrington, M. E. (1997). The ventral lateral geniculate nucleus and the intergeniculate leaflet: interrelated structures in the visual and circadian systems. Neurosci. Biobehav. Rev. 21, 705-727. doi: 10.1016/S0149-7634(96)00019-X

Hattar, S., Kumar, M., Park, A., Tong, P., Tung, J., Yau, K. W., et al. (2006). Central projections of melanopsin-expressing retinal ganglion cells in the mouse. $J$. Comp. Neurol. 497, 326-349. doi: 10.1002/cne.20970

Jagota, A., and Kalyani, D. (2008). Daily serotonin rhythms in rat brain during postnatal development and aging. Biogerontology 9, 229-234. doi: $10.1007 / \mathrm{s} 10522-008-9132-\mathrm{z}$

Jeon, M. H., and Jeon, C. J. (1998). Immunocytochemical localization of calretinin containing neurons in retina from rabbit, cat, and dog. Neurosci. Res. 32, 75-84. doi: 10.1016/S0168-0102(98)00070-4

Khachaturian, Z. S. (1994). Calcium hypothesis of Alzheimer's disease and brain aging. Ann. N. Y. Acad. Sci. 747, 1-11. doi: 10.1111/j.1749-6632.1994.tb44398.x

Kondratova, A. A., and Kondratov, R. V. (2012). Circadian clock and pathology of the ageing brain. Nat. Rev. Neurosci. 13, 325-335. doi: 10.1038/nrn3208

Lamerand, S., Shahidehpour, R., Ayala, I., Gefen, T., Mesulam, M. M., Bigio, E., et al. (2020). Calbindin-D28K, parvalbumin, and calretinin in young and aged human locus coeruleus. Neurobiol. Aging 94, 243-249. doi: 10.1016/j.neurobiolaging.2020.06.006

Landfield, P. W. (1987). 'Increased calcium-current' hypothesis of brain aging. Neurobiol. Aging 8, 346-347. doi: 10.1016/0197-4580(87)90074-1

Langel, J., Ikeno, T., Yan, L., Nunez, A. A., and Smale, L. (2018). Distributions of GABAergic and glutamatergic neurons in the brains of a diurnal and nocturnal rodent. Brain Res. 1700, 152-159. doi: 10.1016/j.brainres.2018.08.019

Lee, C. H., Hwang, I. K., Choi, J. H., Yoo, K. Y., Park, O. K., Huh, S. O., et al. (2010a). Age-dependent changes in calretinin immunoreactivity and its protein level in the gerbil hippocampus. Neurochem. Res. 35, 122-129. doi: 10.1007/s11064-009-0037-2

Lee, E. S., Lee, J. Y., and Jeon, C. J. (2010b). Types and density of calretinincontaining retinal ganglion cells in mouse. Neurosci. Res. 66, 141-150. doi: 10.1016/j.neures.2009.10.008

Lee, S. C. S., Weltzien, F., Madigan, M. C., Martin, P. R., and Grünert, U. (2016) Identification of AII amacrine, displaced amacrine, and bistratified ganglion cell types in human retina with antibodies against calretinin. J. Comp. Neurol. 524, 39-53. doi: 10.1002/cne.23821

Long, J. M., Mouton, P. R., Jucker, M., and Ingram, D. K. (1999). What counts in brain aging? Design-based stereological analysis of cell number. J. Gerontol. A Biol. Sci. Med. Sci. 54, B407-B417. doi: 10.1093/gerona/54.10.B407

López-Otín, C., Blasco, M. A., Partridge, L., Serrano, M., and Kroemer, G. (2013). The hallmarks of aging. Cell 153, 1194-1217. doi: 10.1016/j.cell.2013.05.039

Lucas, R. J., Peirson, S. N., Berson, D. M., Brown, T. M., Cooper, H. M., Czeisler, C. A., et al. (2014). Measuring and using light in the melanopsin age. Trends Neurosci. 37, 1-9. doi: 10.1016/j.tins.2013.10.004

Lukas, W., and Jones, K. A. (1994). Cortical neurons containing calretinin are selectively resistant to calcium overload and excitotoxicity in vitro. Neuroscience 61, 307-316. doi: 10.1016/0306-4522(94)90233-X

Lupi, D., Semo, M., and Foster, R. G. (2012). Impact of age and retinal degeneration on the light input to circadian brain structures. Neurobiol. Aging 33, 383-392. doi: 10.1016/j.neurobiolaging.2010.03.006

Maier, T., Güell, M., and Serrano, L. (2009). Correlation of mRNA and protein in complex biological samples. FEBS Lett. 583, 3966-3973. doi: 10.1016/j.febslet.2009.10.036

Massouh, M., Wallman, M. J., Pourcher, E., and Parent, A. (2008). The fate of the large striatal interneurons expressing calretinin in Huntington's disease. Neurosci. Res. 62, 216-224. doi: 10.1016/j.neures.2008. 08.007 
Matute, C., and Streit, P. (1985). Selective retrograde labeling with D-[3H]aspartate in afferents to the mammalian superior colliculus. J. Comp. Neurol. 241, 34-49. doi: 10.1002/cne.902410104

Melchitzky, D. S., Eggan, S. M., and Lewis, D. A. (2005). Synaptic targets of calretinin-containing axon terminals in macaque monkey prefrontal cortex. Neuroscience 130, 185-195. doi: 10.1016/j.neuroscience.2004.08.046

Míguez, J. M., Recio, J., Sánchez-Barceló, E., and Aldegunde, M. (1998). Changes with age in daytime and nighttime contents of melatonin, indoleamines, and catecholamines in the pineal gland: a comparative study in rat and Syrian hamster. J. Pineal Res. 25, 106-115. doi: 10.1111/j.1600-079X.1998.tb00547.x

Monavarfeshani, A., Sabbagh, U., and Fox, M. A. (2017). Not a one-trick pony: Diverse connectivity and functions of the rodent lateral geniculate complex. Vis. Neurosci. 34, E012. doi: 10.1017/S0952523817000098

Moore, R. Y. (2016). Calretinin neurons in the rat suprachiasmatic nucleus. J. Biol. Rhythms 31, 406-410. doi: 10.1177/0748730416654024

Moore, R. Y., and Speh, J. C. (1993). GABA is the principal neurotransmitter of the circadian system. Neurosci. Lett. 150, 112-116. doi: 10.1016/0304-3940(93)90120-A

Morin, L. P. (2013). Neuroanatomy of the extended circadian rhythm system. Exp. Neurol. 243, 4-20. doi: 10.1016/j.expneurol.2012.06.026

Morin, L. P., and Studholme, K. M. (2014). Retinofugal projections in the mouse. J. Comp. Neurol. 522, 3733-3753. doi: 10.1002/cne.23635

Münkle, M. C., Waldvogel, H. J., and Faull, R. L. (2000). The distribution of calbindin, calretinin and parvalbumin immunoreactivity in the human thalamus. J. Chem. Neuroanat. 19, 155-173. doi: 10.1016/S0891-0618(00)00060-0

Osterhout, J. A., Josten, N., Yamada, J., Pan, F., Wu, S., Nguyen, P. L., et al. (2011). Cadherin-6 mediates axon-target matching in a non-image-forming visual circuit. Neuron 71, 632-639. doi: 10.1016/j.neuron.2011.07.006

Ouda, L., Burianova, J., and Syka, J. (2012). Age-related changes in calbindin and calretinin immunoreactivity in the central auditory system of the rat. Exp. Gerontol. 47, 497-506. doi: 10.1016/j.exger.2012.04.003

Pal, I., Paltati, C. R. B., Kaur, C., Shubhi, S., Kumar, P., Jacob, T. G., et al. (2019). Morphological and neurochemical changes in GABAergic neurons of the aging human inferior colliculus. Hear. Res. 377, 318-329. doi: 10.1016/j.heares.2019.02.005

Paxinos, G., and Watson, C. (2014). The Rat Brain in Stereotaxic Coordinates, 7th Edn. London: Elsevier Academic Press.

Pike, C. J., and Cotman, C. W. (1995). Calretinin-immunoreactive neurons are resistant to beta-amyloid toxicity in vitro. Brain Res. 671, 293-298. doi: 10.1016/0006-8993(94)01354-K

Pillay, S., Bhagwandin, A., Bertelsen, M. F., Patzke, N., Engler, G., Engel, A. K., et al. (2020). The diencephalon of two carnivore species: the feliform banded mongoose and the caniform domestic ferret. J. Comp. Neurol. doi: $10.1002 /$ cne. 25036

Sabbagh, U., Govindaiah, G., Somaiya, R. D., Ha, R. V., Wei, J. C., Guido, W., et al. (2020). Diverse GABAergic neurons organize into subtype-specific sublaminae in the ventral lateral geniculate nucleus. J. Neurochem. 00, 1-19. doi: $10.1111 /$ jnc. 15101

Sabbagh, U., Monavarfeshani, A., Su, K., Zabet-Moghadam, M., Cole, J., Carnival, E., et al. (2018). Distribution and development of molecularly distinct perineuronal nets in visual thalamus. J. Neurochem. 147, 626-646. doi: $10.1111 /$ jnc. 14614

Saiz-Sanchez, D., De la Rosa-Prieto, C., Ubeda-Banon, I., and Martinez-Marcos, A. (2015). Interneurons, tau and amyloid- $\beta$ in the piriform cortex in Alzheimer's disease. Brain Struct. Funct. 220, 2011-2025. doi: 10.1007/s00429-014-0771-3

Saiz-Sanchez, D., Ubeda-Bañon, I., De la Rosa-Prieto, C., and MartinezMarcos, A. (2012). Differential expression of interneuron populations and correlation with amyloid- $\beta$ deposition in the olfactory cortex of an A $\beta P P / P S 1$ transgenic mouse model of Alzheimer's disease. J. Alzheimers Dis. 31, 113-129. doi: 10.3233/JAD-2012-111889

Schurmans, S., Schiffmann, S. N., Gurden, H., Lemaire, M., Lipp, H. P., Schwam, V., et al. (1997). Impaired long-term potentiation induction in dentate gyrus of calretinin-deficient mice. PNAS 94, 10415-10420. doi: 10.1073/pnas.94.19.10415

Sondereker, K. B., Stabio, M. E., and Renna, J. M. (2020). Crosstalk: the diversity of melanopsin ganglion cell types has begun to challenge the canonical divide between image-forming and non-image-forming vision. J. Compa. Neurol. 528, 2044-2067. doi: 10.1002/cne.24873

Tremblay, R., Lee, S., and Rudy, B. (2016). GABAergic interneurons in the neocortex: from cellular properties to circuits. Neuron 91, 260-292. doi: 10.1016/j.neuron.2016.06.033

Villa, A., Podini, P., Panzeri, M. C., Racchetti, G., and Meldolesi, J. (1994). Cytosolic $\mathrm{Ca}^{2+}$ binding proteins during rat brain ageing: loss of calbindin and calretinin in the hippocampus, with no change in the cerebellum. Eur. J. Neurosci. 6, 1491-1499. doi: 10.1111/j.1460-9568.1994.tb01010.x

Vishnyakova, P. A., Moiseev, K. Y., Spirichev, A. A., Emanuilov, A. I., Nozdrachev, A. D., and Masliukov, P. M. (2020). Expression of calbindin and calretinin in the dorsomedial and ventromedial hypothalamic nuclei during aging. Anat. Rec. 1-11. doi: 10.1002/ar.24536

von Bartheld, C. S., von, Bahney, J., and Herculano-Houzel, S. (2016). The search for true numbers of neurons and glial cells in the human brain: a review of 150 years of cell counting. J. Comp. Neurol. 524, 3865-3895. doi: 10.1002/cne.24040

West, M. J., Slomianka, L., and Gundersen, H. J. (1991). Unbiased stereological estimation of the total number of neurons in thesubdivisions of the rat hippocampus using the optical fractionator. Anat. Rec. 231, 482-497. doi: 10.1002/ar.1092310411

Yan, F. F., Hou, F., Lu, H., Yang, J., Chen, L., Wu, Y., et al. (2020). Aging affects gain and internal noise in the visual system. Sci. Rep. 10:6768. doi: 10.1038/s41598-020-63053-0

Conflict of Interest: The authors declare that the research was conducted in the absence of any commercial or financial relationships that could be construed as a potential conflict of interest.

Copyright (C) 2021 Fiuza, Queiroz, Aquino, Câmara, Brandão, Lima, Cavalcanti, Engelberth and Cavalcante. This is an open-access article distributed under the terms of the Creative Commons Attribution License (CC BY). The use, distribution or reproduction in other forums is permitted, provided the original author(s) and the copyright owner(s) are credited and that the original publication in this journal is cited, in accordance with accepted academic practice. No use, distribution or reproduction is permitted which does not comply with these terms. 